\title{
Different Synchronization Schemes for Chaotic Rikitake Systems
}

\author{
Mohammad Ali Khan \\ Department of Mathematics, Garhbeta Ramsundar Vidyabhaban, \\ West Bengal, India \\ E-mail: mdmaths@gmail.com
}

\begin{abstract}
This paper presents the chaos synchronization by designing different type of controller. Firstly, we propose the synchronization of bidirectional coupled chaotic Rikitake systems via hybrid feedback control. Secondly, we study the synchronization of unidirectionally coupled Rikitake systems using hybrid feedback control. Lastly, we investigate the synchronization of unidirectionally coupled Rikitake chaotic systems using tracking control. Comparing all results finally we conclude that tracking control is more effective than feedback control. Simulation results are presented to show the efficiency of synchronization schemes.
\end{abstract}

Keywords: Chaotic system, Chaos synchronization, Chaos control, Rikitake system.

\section{Introduction}

Since the pioneer works by Ott.E, Grebogi and Yorke [1] and Pecorra and Carroll [2] , chaos control and synchronization has received increasing attention due to its theoretical challenges and its potential applications to various disciplines. Synchronization in biological systems is one of the fascinating area that has attracted a lots of renewed attention.

Historically, the analysis of synchronization phenomena in the evaluation dynamical system has been a subject of active investigation since the earlier days of physics. Chaos synchronization become very important topics in the non-linear science over the last two decades, due to its potential applications in many areas 
such as secure communication, information processing, biological system, chemical reaction, neural networks and in engineering. Usually two dynamical systems are called synchronized if the distance between their corresponding states converges to zero as time goes to infinity. This type of synchronization is known as identical synchronization [1]. Using linear and non linear feedback control chaos synchronization have been presented in various chaotic systems. Synchronization of unified chaotic system using adaptive feedback control was studied by Lu and Chen in 2002 [3]. In 2005, Park [4] has studied controlling chaotic systems via nonlinear feedback control. Chen et.al. [5] proposed generating hyper chaotic Lu attractor via state feedback control. Synchronization between two different noise perturbed chaotic system with fully unknown parameters was proposed by Sun Y.Cao in 2007 [6]. Poria et.al. [7] have investigated adaptive synchronization of two coupled chaotic Neuronal systems. Khan et.al. [8] have investigated control strategies for unified chaotic system using different type of control. Recently in 2009 Tarai et.al. $[9,10]$ have observed generalized synchronization of bidirectionally coupled chaotic system .

In this paper firstly, we discuss the synchronization between two bidirectionally coupled chaotic Rikitake system via hybrid feedback control. Secondly, we study the synchronization between two identical Rikitake system using hybrid feedback control and lastly, we investigates the synchronization of two identical Rikitake chaotic systems using tracking control.

\section{Design of Hybrid Controller of Bidirectionally Couple Chaotic System}

A dynamical system can be written as

$$
\dot{x}=f(x)
$$

This system can also be expressed as

$$
\dot{x}=A x+B \Psi(x)
$$

where $A \in R^{n \times n}, B \in R^{n \times m}$ are constant matrix, $\Psi(x): R^{n} \rightarrow R^{n}$ is non-linear vector functions. We consider the following type bidirectionally coupled chaotic systems

$$
\begin{aligned}
& \dot{x}=A x+B\left[\Psi(x)+u_{1}\right] \\
& \dot{y}=A y+B\left[\Psi(y)+u_{2}\right]
\end{aligned}
$$

where $x \in R^{n}, y \in R^{n}, A \in R^{n \times n}, B \in R^{n \times m}, \Psi: R^{n} \rightarrow R^{n}$ is non-linear vector functions. The synchronization errors between the systems (3) and (4) are defined as $e=\left(e_{1}, e_{2}, \ldots \ldots . ., e_{n}\right)^{T}=\left(x_{1}-y_{1}, x_{2}-y_{2}, \ldots . ., x_{n}-y_{n}\right)^{T}$. Then the error dynamical system is 


$$
\dot{e}=A e+B\left[\Psi(x)-\Psi(y)+u_{1}-u_{2}\right]
$$

In order to make the system (3) and (4) synchronizable, the coupling functions $u_{1}$ and $u_{2}$ should be properly chosen.

Let $u_{1}=u_{11}+u_{12}$ and $u_{2}=u_{21}+u_{22}$, where $u_{11}=\Psi(y), u_{12}=-K y$ and $u_{21}=\Psi(x), u_{22}=-K x$ and $K=\left(\begin{array}{ccc}k_{1} & 0 & 0 \\ 0 & k_{2} & 0 \\ 0 & 0 & k_{3}\end{array}\right)$, denote the feedback matrix.

Obviously $u_{11}, u_{21}$ are non-linear controller and $u_{12}, u_{22}$ are linear controller, so $u_{1}$ and $u_{2}$ are hybrid controller.

Theorem: If the matrix $A+B K$ has all eigen values with negative real parts, then origin will be a asymptotically stable fixed point of the system (5).

Proof: Choosing the controller $u_{1}$ and $u_{2}$ properly, the error system (5) can be written as

$$
\dot{e}=(A+B K) e
$$

Now by the theory of linear dynamical system if the matrix $A+B K$ has all eigen values with negative real parts, then the origin of the error system will be globally asymptotically stable fixed point. Therefore in this case the bi-directionally coupled systems (3) and (4) will synchronize.

Example. We shall now discuss efficiency of our scheme taking coupled chaotic Rikitake systems. The Rikitake system can be described by the following system of differential equations.

$$
\begin{aligned}
& \dot{x}_{1}=x_{2} x_{3}-a_{2} x_{1} \\
& \dot{x}_{2}=\left(x_{3}-b_{2}\right) x_{1}-a_{2} x_{2} \\
& \dot{x}_{3}=1-x_{1} x_{2}
\end{aligned}
$$

where $x_{1}, x_{2}, x_{3} \in R^{n}$ are state variables and $a_{2}, b_{2}$ are real constants. System (7) is found to be chaotic when $a_{2}=2$ and $b_{2}=5$.

According to our choice of controller the coupled systems are

$$
\begin{aligned}
& \dot{x}_{1}=x_{2} x_{3}-a_{2} x_{1}+y_{2} y_{3}-k_{1} y_{1} \\
& \dot{x}_{2}=\left(x_{3}-b_{2}\right) x_{1}-a_{2} x_{2}+y_{1} y_{3}-k_{2} y_{2} \\
& \dot{x}_{3}=2-x_{1} x_{2}-y_{1} y_{2}+y_{3}-k_{3} y_{3}
\end{aligned}
$$

and 


$$
\begin{aligned}
& \dot{y}_{1}=y_{2} y_{3}-a_{2} y_{1}+x_{2} x_{3}-k_{1} x_{1} \\
& \dot{y}_{2}=\left(y_{3}-b_{2}\right) y_{1}-a_{2} y_{2}+x_{1} x_{3}-k_{2} x_{2} \\
& \dot{y}_{3}=2-y_{1} y_{2}-x_{1} x_{2}+x_{3}-k_{3} x_{3}
\end{aligned}
$$

Therefore the error system is

$$
\begin{aligned}
& \dot{e_{1}}=-\left(a_{2}-k_{1}\right) e_{1} \\
& \dot{e_{2}}=-b_{2} e_{1}+\left(k_{2}-a_{2}\right) e_{2} \\
& \dot{e_{3}}=\left(k_{3}-1\right) e_{3}
\end{aligned}
$$

Now for suitable choice of $K$ the matrix $A+B K$ has all eigen values with negative real parts, then the drive system synchronizes with the response system.

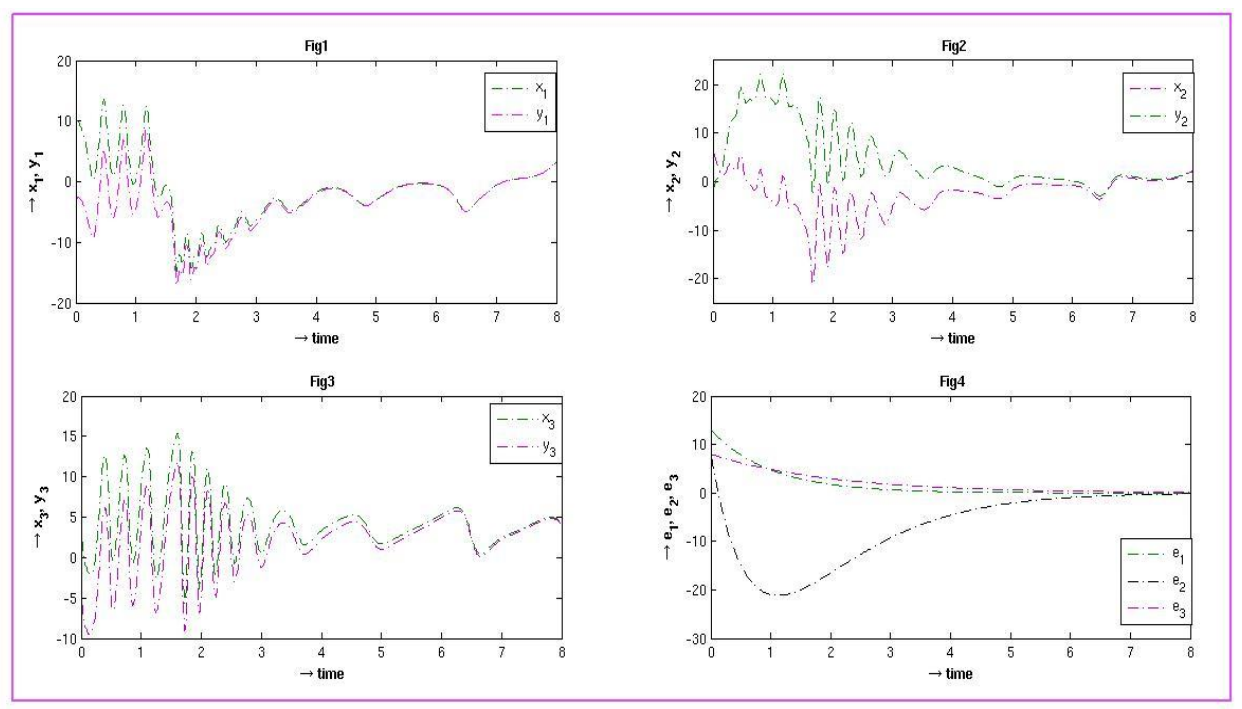

Fig.1-Fig.3. represents the trajectories of $\left(\mathrm{x}_{1}, \mathrm{y}_{1}\right),\left(\mathrm{x}_{2}, \mathrm{y}_{2}\right)$ and $\left(\mathrm{x}_{3}, \mathrm{y}_{3}\right)$ and Fig.4. shows time evolution of the synchronization error.

We choose $A=\left(\begin{array}{ccc}-2 & 0 & 0 \\ -5 & -2 & 0 \\ 0 & 0 & -1\end{array}\right), B=\left(\begin{array}{ccc}1 & 0 & 0 \\ 0 & 1 & 0 \\ 0 & 0 & 1\end{array}\right)$, and $K=\left(\begin{array}{ccc}1 & 0 & 0 \\ 0 & 1 & 0 \\ 0 & 0 & .5\end{array}\right)$.

For numerical simulation, Fourth order Runge-Kutta method is used. We select the parameters $\left(a_{2}, b_{2}\right)=(2,5)$. The initial conditions of system (8) and (9) are chosen as $\left(x_{1}(0), x_{2}(0), x_{3}(0)\right)=(10,6,3)$ and $\left(y_{1}(0), y_{2}(0), y_{3}(0)\right)=(-3,-2,-5)$, so the initial synchronization errors are $\left(e_{1}(0), e_{2}(0), e_{3}(0)\right)=(13,8,8)$. The trajectories of the $x_{1}$, state of the drive system and $y_{1}$, state of the response system are shown in Fig.1. The trajectories of $x_{2}$ and $y_{2}$ are shown in Fig.2. and the trajectories of $x_{3}$ and $y_{3}$ are shown in Fig.3. Figures confirm the synchronization between the two chaotic bidirectionally systems. Time evolution of the synchronization error goes to zero which are shown in Fig.4. 


\section{Synchronization of Coupled Rikitake Systems Via Hybrid Feedback Control}

In this section, we discuss the synchronization of coupled Rikitake systems via hybrid feedback control. The Rikitake system (7) can be rewritten as

$$
\left(\begin{array}{c}
\dot{x}_{1} \\
\dot{x}_{2} \\
\dot{x}_{3}
\end{array}\right)=\left(\begin{array}{ccc}
-a_{2} & 0 & 0 \\
-b_{2} & -a_{2} & 0 \\
0 & 0 & -1
\end{array}\right)\left(\begin{array}{l}
x_{1} \\
x_{2} \\
x_{3}
\end{array}\right)+\left(\begin{array}{ccc}
1 & 0 & 0 \\
0 & 1 & 0 \\
0 & 0 & 1
\end{array}\right)\left(\begin{array}{c}
x_{2} x_{3} \\
x_{1} x_{3} \\
1+x_{3}-x_{1} x_{2}
\end{array}\right)
$$

Comparing equation (3.1) with equation (2.2), we get

$$
\begin{gathered}
A=\left(\begin{array}{ccc}
-a_{2} & 0 & 0 \\
-b_{2} & -a_{2} & 0 \\
0 & 0 & -1
\end{array}\right), B=\left(\begin{array}{ccc}
1 & 0 & 0 \\
0 & 1 & 0 \\
0 & 0 & 1
\end{array}\right), \Psi(x)=\left(\begin{array}{c}
x_{2} x_{3} \\
x_{1} x_{3} \\
1+x_{3}-x_{1} x_{2}
\end{array}\right) \\
\text { Now, } A-B K=\left(\begin{array}{ccc}
-2-k_{1} & 0 & 0 \\
-5 & -2-k_{2} & 0 \\
0 & 0 & -1-k_{3}
\end{array}\right) \text { where } a_{2}=2 \text { and } b_{2}=5 .
\end{gathered}
$$

The characteristic equation of $A-B K$ is

$$
\lambda^{3}+\lambda^{2}\left(k_{1}+k_{2}+k_{3}+5\right)+\lambda\left(k_{1} k_{2}+k_{1} k_{3}+k_{2} k_{3}+3 k_{1}+3 k_{2}+4 k_{3}+8\right)+\left(k_{1}+2\right)\left(k_{2}+2\right)\left(k_{3}+1\right)=0
$$

According to Routh-Hurwitz, the matrix $A-B K$ is negative definite if

$$
\begin{aligned}
& k_{1}+k_{2}+k_{3}+5>0 \\
& k_{1} k_{2}+k_{1} k_{3}+k_{2} k_{3}+3 k_{1}+3 k_{2}+4 k_{3}+8>0 \\
& \left(k_{1}+2\right)\left(k_{2}+2\right)\left(k_{3}+1\right)>0 \\
& \left(k_{1}+k_{2}+k_{3}+5\right)\left(k_{1} k_{2}+k_{1} k_{3}+k_{2} k_{3}+3 k_{1}+3 k_{2}+4 k_{3}+8\right)>\left(k_{1}+2\right)\left(k_{2}+2\right)\left(k_{3}+1\right)
\end{aligned}
$$

are satisfied when $k_{1}=1, k_{2}=1$ and $k_{3}=.5$ then $A-B K$ is negative definite.

Therefore the controller $u_{1}$ and $u_{2}$ can be chosen as

$$
u_{1}=\left(\begin{array}{c}
x_{2} x_{3}-y_{2} y_{3} \\
x_{1} x_{3}-y_{1} y_{3} \\
-x_{1} x_{2}+y_{1} y_{2}+x_{3}-y_{3}
\end{array}\right) \text { and } u_{2}=K(x-y)=\left(\begin{array}{ccc}
k_{1} & 0 & 0 \\
0 & k_{2} & 0 \\
0 & 0 & k_{3}
\end{array}\right)\left(\begin{array}{c}
x_{1}-y_{1} \\
x_{2}-y_{2} \\
x_{3}-y_{3}
\end{array}\right)
$$

Therefore the response system becomes 


$$
\begin{aligned}
& \dot{y}_{1}=-\left(a_{2}+1\right) y_{1}+x_{1}+x_{2} x_{3} \\
& \dot{y}_{2}=-b_{2} y_{1}-\left(a_{2}+1\right) y_{2}+x_{2}+x_{1} x_{3} \\
& \dot{y}_{3}=\frac{1}{2}\left(x_{3}-y_{3}\right)-x_{1} x_{2}+1
\end{aligned}
$$

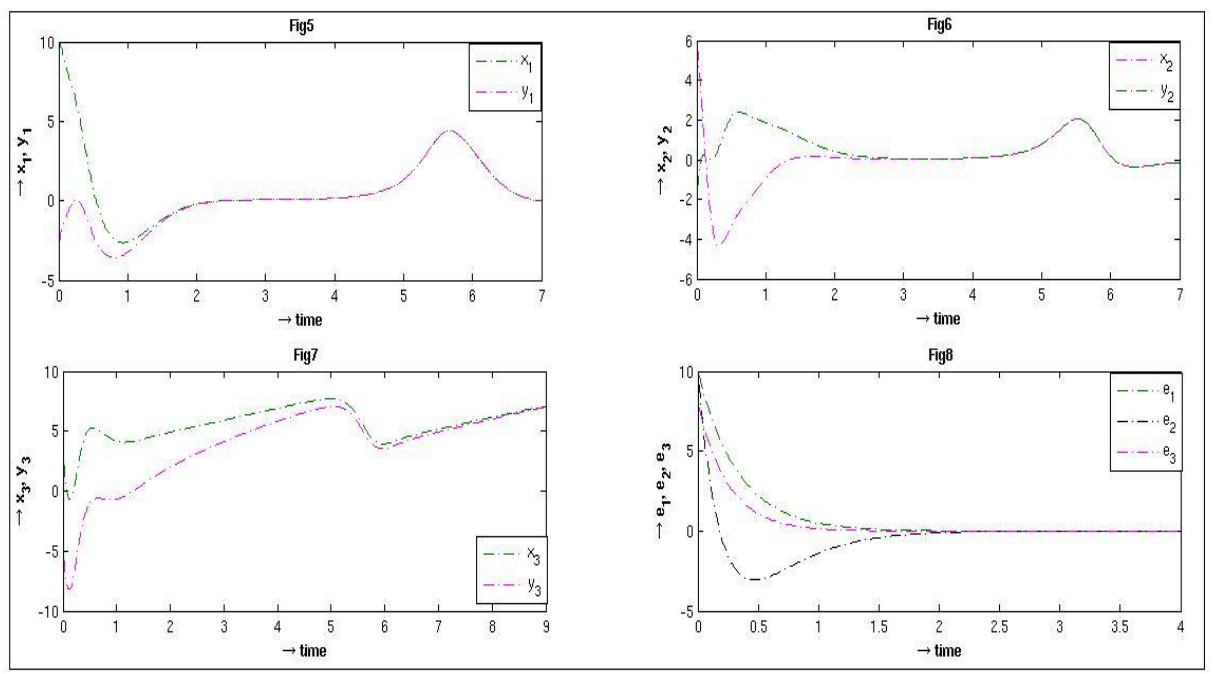

Fig.5-Fig.7. represents the trajectories of $\left(\mathrm{x}_{1}, \mathrm{y}_{1}\right),\left(\mathrm{x}_{2}, \mathrm{y}_{2}\right)$ and $\left(\mathrm{x}_{3}, \mathrm{y}_{3}\right)$ and Fig.8. shows time evolution of the synchronization error.

For numerical simulation,we select the parameter $\left(a_{2}, b_{2}\right)=(2,5)$. The initial conditions of system (11) and (13) are chosen as $\left(x_{1}(0), x_{2}(0), x_{3}(0)\right)=(10,6,3)$ and $\left(y_{1}(0), y_{2}(0), y_{3}(0)\right)=(-3,-2,-5)$, so the initial values of errors are $(13,8,8)$. The trajectories of $x_{1}$, state of the drive system and $y_{1}$, state of the response system are shown in Fig.5. The trajectories of $x_{2}$ and $y_{2}$ are shown in Fig.6. and the trajectories of $x_{3}$ and $y_{3}$ are shown in Fig.7. Figures confirm the synchronization behavior between the coupled systems. Time evolution of the synchronization errors converges to zero which are shown in Fig.8.

\section{Design of Tracking Controller}

For discuss the synchronization of unidirectional coupled chaotic system via tracking control we assume that system (2) is the drive system. By introducing control variable $U \in R^{n}$, then the controlled response system is given by

$$
\dot{y}=A y+B \Psi(y)+U
$$

where $y \in R^{n}$ denote the state vector of the response system. The main problem is 
to design a controller $U$ which synchronizes the state of both drive and response systems. We substract (2) from (14) and we get

$$
\dot{e}=A e+B(\Psi(y)-\Psi(x))+U
$$

where $e=y-x$. The aim is to make $\lim _{t \rightarrow \infty}\|e(t)\|=0$. Let the Lyapunov error function be $V(e)=\frac{1}{2} e^{T} e$, where $V(e)$ is a positive definite function. Assuming that the parameters of the drive and response systems are known and state of both system are measurable. We may achieve the synchronization by selecting the controller $U$ to make the first derivative $V(e)$ i.e. $\& e)<0$. Then the state of the response and drive system synchronized asymptotically globally.

\section{Example:}

Synchronization of Rikitake system via tracking control

Let dynamical system (7) be the drive system, then the controlled response Rikitake system is given by the following

$$
\begin{aligned}
& \dot{y}_{1}=y_{2} y_{3}-a_{2} y_{1}+u_{1} \\
& \dot{y}_{2}=\left(y_{3}-b_{2}\right) y_{1}-a_{2} y_{2}+u_{2} \\
& \dot{y}_{3}=1-y_{1} y_{2}+u_{3}
\end{aligned}
$$

Let us define the error between the trajectories of the response and drive Rikitake system as $e_{i}=y_{i}-x_{i} \quad(i=1,2,3)$. Therefore the error system is

$$
\begin{aligned}
& \dot{e}_{1}=-a_{2} e_{1}+y_{2} y_{3}-x_{2} x_{3}+u_{1} \\
& \dot{e}_{2}=-b_{2} e_{1}-x_{1} x_{3}+y_{1} y_{3}-a_{2} e_{2}+u_{2} \\
& \dot{e}_{3}=x_{1} x_{2}-y_{1} y_{2}+u_{3}
\end{aligned}
$$

If we choose the controller as

$$
\begin{aligned}
& u_{1}=x_{2} x_{3}-y_{2} y_{3} \\
& u_{2}=x_{1} x_{3}-y_{1} y_{3}+b_{2} e_{1} \\
& u_{3}=y_{1} y_{2}-x_{1} x_{2}-e_{3}
\end{aligned}
$$

Then the synchronization between the coupled Rikitake systems will occur. 


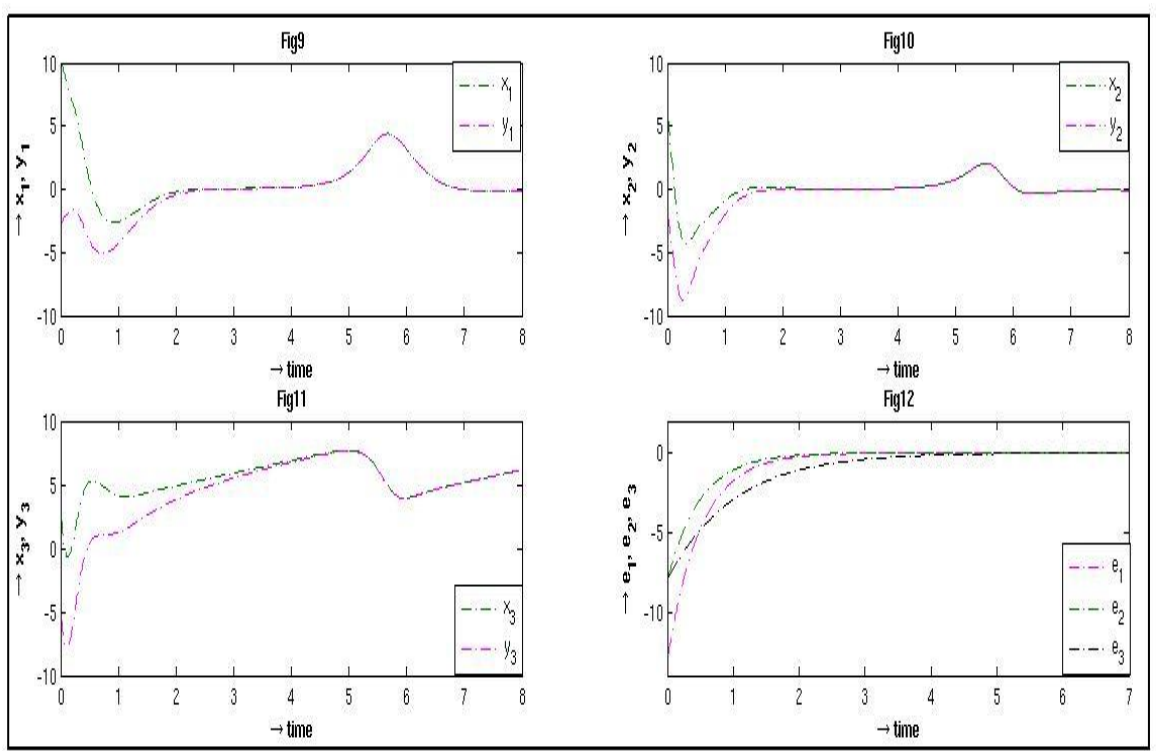

Fig.9-Fig.11.represents the trajectories of $\left(\mathrm{x}_{1}, \mathrm{y}_{1}\right),\left(\mathrm{x}_{2}, \mathrm{y}_{2}\right)$ and $\left(\mathrm{x}_{3}, \mathrm{y}_{3}\right)$ and Fig.12. shows time evolution of the synchronization errors.

The parameters of Rikitake system are $\left(a_{2}, b_{2}\right)=(2,5)$. The initial conditions for the driving system and driven system are given respectively by $\left(x_{1}(0), x_{2}(0), x_{3}(0)\right)=(10,6,3)$ and $\left(y_{1}(0), y_{2}(0), y_{3}(0)\right)=(-3,-2,-5)$, so the initial values of error system are $\left(e_{1}(0), e_{2}(0), e_{3}(0)\right)=(13,8,8)$. The trajectories of $x_{1}$, state of the drive system and $y_{1}$, state of the response system are shown in Fig.9. The trajectories of $x_{2}$ and $y_{2}$ are shown in Fig.10. and the trajectories of $x_{3}$ and $y_{3}$ are shown in Fig.11. Figures confirm the synchronization behavior between the system. Time evolution of the synchronization errors go to zero which are shown in Fig. 12.

\section{Conclusions}

This paper investigates the synchronization by designing different type controller, which include bi-directional coupled chaotic systems using hybrid feedback control and unidirectional coupled chaotic systems using hybrid feedback control and tracking control. We apply above three controller methods for Rikitake system to prove the feasibility and effectiveness of the proposed scheme. Comparing all results finally we conclude from numerical simulation results that tracking control is more effective than feedback control. 


\section{Acknowledgement}

I acknowledge Dr. Swarup Poria, Department of Applied Mathematics, Calcutta

University, Kolkata for his useful suggestions for preparation of this paper.

\section{References}

[1] E. Ott, C. Grebogi and J.A. Yorke, Controlling chaos, Phys. Rev.Lett., 64 (1990)1196-1199.

[2] L.M. Pecora and T.L. Carroll, Synchronization in chaotic systems, Phys. Rev. Lett., 64 (1990) 821-824.

[3] S. Chen and J. Lu, Synchronization of an uncertain unified chaotic system via adaptive control, Chaos, Solitons and Fractals, 14(2002) 643-647.

[4] J.H. Park, Controlling chaotic systems via nonlinear feedback control, Chaos, Solitons and Fractals, 23(2005) 1049-1054.

[5] A.M. Chen, J.N. Lu and J.H. Lii, Generating hyperchaotic Lu attractor via state feedback control, Physics A, 364(2006) 103-110.

[6] Y. San and J. Cao, Adaptive synchronization between two different noiseperturbed chaotic system with fully unknown parameters, Physica A, 376(2007) 253-265.

[7] S. Poria and A. Tarai, Adaptive synchronization of two coupled chaotic Neuronal systems, Rev.Bull.Cal.Math.Soc., 15(2007)53-60.

[8] M. Khan, A.Mondal and S.Poria, Three control strategies for unified chaotic system, Int. J. of App. Mech. and Eng., 16(2011)597-608.

[9] A Tarai(Poria), S Poria and P. Chatterjee, Synchronization of bidirectionally coupled chaotic Chen's system with delay, Chaos Solitons and Fractals, 41 (2009) 643-647.

[10] A. Tarai, S.Poria and P. Chatterjee, Synchronization of generalized linearly bidirectionally coupled unified system, Chaos Solitons and Fractals. 40 (2009) 885-892. 